\title{
The object of art in science: science communication via art installation
}

\author{
Patricia Rios and Aquiles Negrete Yankelevich
}

\begin{abstract}
Science is part of our everyday live; so is art. Some art installations that link the two require the active presence of the spectator. Thereby they help to raise the awareness, promote understanding, and generate an emotional response from the public. This project rests on the public participation model that seeks to explore the connection between art installations and science communication through experiential learning. In order to test the effectiveness of an art installation communicating science two groups were contrasted. The first was exposed to a list of scientific facts; the second participated in the creation of an art installation. The results of this research suggest that art installations do promote long-term fact retention. Therefore, the use of art installations can be considered an interesting method of conveying science in an attractive, reliable, and memorable way.
\end{abstract}

\section{Introduction}

Science communicators constantly struggle to achieve a better diffusion of scientific knowledge. The public is bombarded with information in ways that are not necessarily attractive and/or understandable. It becomes discouraged in its attempt to penetrate the scientific world and erroneously ends up avoiding the topic. Consequently, science communicators wonder how to foster the general public's interest in scientific knowledge. How to construct a bridge between science and society in general? How to make scientific knowledge (not only technology) part of our culture and daily life?

It is worth noting that in recent years, science communication has become a multidirectional, inter-disciplinary process, in which different disciplines (sciences and other knowledge areas) overlap and interact in order to attempt to provide an account for the world in which we live. Identifying possible interactions among diverse disciplines might provide the means to communicate scientific knowledge in a successful way. This research explores a method of art expression that has been rising in recent years and which involves public participation. It has been shown to successfully engage people from different backgrounds and age groups.

In an attempt to contribute to bridge the gap between science and the public this work explores the possibility of bringing together science and art installations (the well known two C.P Snow ${ }^{1}$ cultures) as a means of conveying scientific knowledge in an attractive, understandable and memorable way. It uses the Retell, Identify, Remember, 
Contextualize (RIRC) method (a method that assesses effectiveness communication using narratives) in order to provide qualitative evidence of the success of communication. The following diagram conceptualizes this research's structure (figure 1).

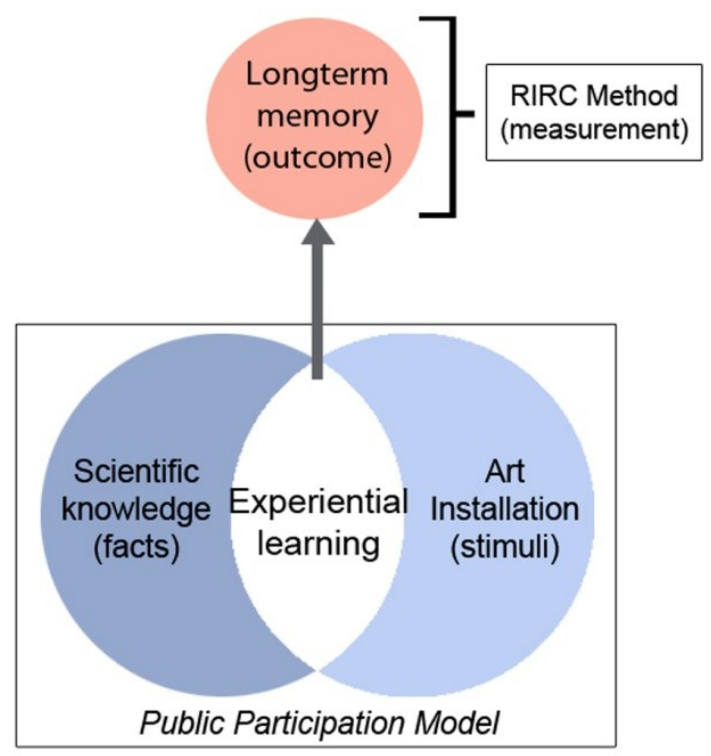

Figure 1. The research structure brings together scientific facts and the art installation through experiential learning.

\section{The public participation model}

There are various ways in which the public can be actively involved and participate in science and technology. The lowest and most simple level, in which knowledge moves over a one-way communication pathway going from the scientific community to the public, requires a low level of involvement as well as little awareness or understanding. The highest level of participation, and most complex one, requires more knowledge on the part of the public and a dialogue between the parties in order to

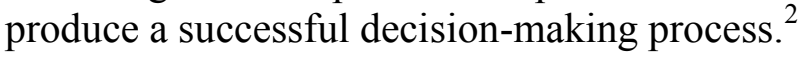

Indeed, it is important that scientists learn how to effectively communicate their knowledge to the general public, but it is also important to develop the public's understanding of science so the public can be part of the highest level of the decisionmaking process. ${ }^{3}$ However, one of the most important issues that should be addressed is how to engage the public in order to develop a sense of responsibility, awareness, and belonging. The public's input will only become compelling when it is engaged and truthfully cares about how its decisions will affect its future.

There is evidence that, in museums and science centers, interactive science exhibitions (a similar experience to the art installation) represent a successful tool to convey scientific knowledge in an enjoyable, reliable and memorable way. ${ }^{4}$ By 
implementing art installation as a tool for science communication, this research aims to focus on both, the public and the scientific knowledge; to make scientific knowledge more meaningful and memorable and to create awareness on the public on scientific and technological issues.

\section{Art installations}

Art, specifically postmodern contemporary art, is starting to take hold as a learning tool in science education and communication. It is implemented in various ways. Nevertheless, there is a strong tendency to involve feelings, emotions, creativity, and knowledge in order to reinforce cognitive learning. ${ }^{5}$ Art motivates the participant to learn and helps him or her to develop higher-order thinking skills. ${ }^{6}$

Art is valuable since it encourages different forms of human intelligence; it can fulfill the purpose of communicating and entertaining at the same time. ${ }^{7}$ The creation of art results in the integration of information with the imagination. This enables the audience to conceptualize the represented reality.

Artistic installation is a contemporary art genre that began to take a strong hold in the artistic community in 1970. The installations incorporate many artistic mediums to create an emotional or conceptual experience in particular environments. In general, installation artists incorporated the demonstration space as another element of their work: such as public spaces, museums, art galleries, and even urban spaces. ${ }^{8}$

An art installation is an expression performed as a component of a multidisciplinary and multisensory approach. This communication instrument not only speaks to our eyes or intellect, but also through tactile and kinesthetic sensations. ${ }^{9}$ In this kind of work both creativity and its ultimate expression are part of the technique. A strong sensory experience is created with the active presence and participation of the spectator. We are interested in this active presence as a participatory element in the communication of scientific topics. For a long time science lived behind laboratory walls, but nowadays, it is not only necessary for people to understand science, but to seize it and to experience it in order to be able to express an opinion regarding its role in everyday life. ${ }^{10}$

The importance of using artistic installations as a means for science communication is based upon two elements. First, it turns the public into an active participant in the creation and conceptualization of the work of art; therefore the public acquires experiential learning. The flexibility characterized by artistic installations permits themes or topics to be expressed in different forms, textures, sounds, sizes, smells and colors, emphasizing the importance of a sensory approach. ${ }^{11}$ In this way multisensory learning is adapted and internalized by the participant.

Second, scientific communication (SciCom) can be defined as the use of skills, means, activities, and dialogues adapted to produce one or more of the following personal responses towards science: Awareness, Enjoyment, Interest, Opinion-forming and Understanding (AEIOU approach). ${ }^{12}$ The communication of science through artistic installation seeks to incorporate the five components of the AEIOU approach through a process of active participation in a science communication intervention that has been conceptualized in an artistically-designed presentation. 
In a spirit related to the AEIOU approach, linking scientific knowledge and art installation might lead to the creation of a memorable experience that enhances the retention of scientific information in the long-term memory.

\section{Experiential learning}

Experiential learning appeals to one of the most important methods of knowledge acquisition, one in which sensory experience, imagination, and long-term memory construction, play a pivotal role.

According to Aristotle, one's first experience revolves around the senses. Later on, a process of repetition and abstraction of ideas is carried out, in which once having interacted with the images that are created by the imagination, sensory experience becomes intelligible to create knowledge. One of the advantages of experiential learning is that it achieves not only short-term, but long-term knowledge acquisition.

The cognitive process involves making connections, exploring patterns and capturing the "big picture" with all its details. One of the elements that help the memory to retain information is the mnemonic system. This system uses the process of the mental association of ideas. It involves schemes, systematic exercises, and repetitions in order to facilitate the process of memorization. ${ }^{13}$ The mnemonic system also uses visual aids to establish associations that are easy to remember. We often discover that mental images last longer than facts. The ability of the human mind to remember images is generally better than that of remembering words. Imagination is the way to mentally represent a sensation; memory is the way to retain it. As Einstein once said, "Visual images are the antechamber of words."

The psychologist Carl Rogers distinguishes two different types of learning: the cognitive (insignificant) and the experiential (significant). The first corresponds to academic knowledge such as the learning of spelling lists or multiplication tables. The second refers to applied knowledge: such as learning about motors in order to repair an automobile. The key to the distinction is that experiential learning takes into account the needs and desires of the public. ${ }^{14}$

This type of communication seeks to use metaphors that conceptualize scientific knowledge in objects, materials, sounds, and different kind of external stimuli. Art expressions (particularly contemporary art expressions) construct a bridge between imagination and the world's critical issues. In this case, the topic of environmental deterioration is conceptualized as an art installation that will allow participants to capture and understand the importance of this issue through objects, materials, sounds, etc. The stimuli foster the opinion-forming process in the individual participant.

Since the participant is exposed to an actual physical experience, the scientific facts (every time that are remembered) will be related to a life experience or episode. An episodic memory consists of memories that come from different areas of the brain that are bound together to create an episode. This is the type of memory used to remember the events in our lives. ${ }^{15}$ Therefore, it is reasonable to expect that the experiential learning (located in an episode) will produce AEIOU responses in the individual and therefore will enhance the retention of information in one's long term memory. 


\section{Long-term potentiation and memory}

The principal function of long-term potentiation is to enhance the synaptic efficiency that causes memory storage. It may increase and strengthen the effect of external stimuli and therefore facilitate the creation of memories. ${ }^{16}$

Two of the most important processes that allow long-term potentiation activity are associativity and cooperativity. They have been known to foster the learning process. These terms allude to stimuli or events that occur at the same time, and therefore become associated with one another. This is important since events' association can create relevant levels of stimulation that induce long-term potentiation. ${ }^{17}$

Therefore, in order to propose new means to effectively communicate scientific knowledge, it is important to bring together powerful stimuli that have the capacity to reinforce the participant's experience, enhancing long-term potentiation. In this research, the association of events is achieved through a memorable experience of creating an art installation; this includes manipulating objects, being exposed to different odours, colours and forms. In this case information and objects are linked together in order to create a powerful stimulus. Long-term potentiation becomes a key element to be observed in order to communicate scientific facts that are not only important to be understood but also to be remembered as part of the learning process.

In order to measure the effects of participating in an art installation in the retention of scientific information in long-term memory, the RIRC method ${ }^{18}$ was employed to evaluate the comprehension and retention of scientific knowledge.

\section{RIRC method}

The RIRC method is applied through a questionnaire that includes three tasks to measure explicit memory (which involves a conscious recollection of data): declarative knowledge, recognition, and recall. The method also includes a task that measures implicit memory (which involves the use of previous experiences that are not consciously recollected). This is referred as procedural knowledge.

Declarative knowledge refers to recalled facts; recognition implies identifying elements that were previously learned; recall is about producing a fact, a cluster of words, or a story that has been retained in memory. Finally, the tasks that involve procedural knowledge are those in which abilities or behaviors are learned and can be remembered. These groups of memory tasks were designed in order to measure how the public learns and retains information, as well as the different levels of understanding the provided information. ${ }^{19}$

The RIRC method was originally used to compare and contrast the performance of a public exposed to scientific facts using narrative forms. Due to the characteristics of an art installation, the use of different forms, odors and materials, helps create different ways of understanding and perceiving the information. The component regarding Opinion-forming (as represented by the "O" in the AEIOU approach) differs from participant to participant and it is important to analyze the way in which consciousness is address by each one of them. The art installation has an extra task involving 
procedural knowledge that exists in order to understand the relation established between facts and the art installation concepts.

\section{Objectives}

1. To explore the capabilities of art installations with respect to the communication of scientific knowledge.

2. To evaluate how participants understand and remember scientific information from an art installation as compared to a list of scientific facts.

\section{Methodology}

\section{Designing an art installation}

This study was completed as part of two larger projects. The first one seeks to involve students enrolled in the Contemporary Art course in an active representation of one of the genres studied throughout the term. The second and most important project was developed by $\mathrm{PhD}$ students and professors. Its purpose was to explore different cultural media and their effectiveness to convey scientific knowledge. The art installation brings together course material with previous studies that consider art installations as interesting media to communicate science.

Science textbooks have held a privileged position over other media in science education. ${ }^{20}$ However, science and technology are represented in many other forms of communication such as radio, television, news, magazines, music, cinema, art installations and narratives. ${ }^{21 ; 22 ; 23 ; 24}$ If we are to educate society in and about science, as Nunan and Homer ${ }^{25}$ propose, we have to consider all of science's cultural media. They are all cultural expressions of science in our society, as well as receptacles of scientific knowledge and important resources for science communication and education.

As part of our research on science communication via cultural media we decided to explore the capabilities of an art installation to communicate science.

The art installation was previously designed by a group of experts in the subject of science communication and contemporary art. Several proposals for the art piece were presented before deciding on the art installation that would require the public's active participation and include different objects, colors, and odors to satisfy the premises of experiential learning.

The authors studied the most representative examples of art installations and alternative cultural media to communicate science. For the art installations, they selected the most representative artistic interventions that allow the public to interact with the art piece through a memorable experience. From the following art installations three notions were highlighted in order to create the installation: a powerful image, process of transformation, and publics' role in participation.

- "Head on" by Cai Guo Qiang. The author represents the history of Berlin and the destiny of humankind. It was conceptualized by a pack of ninety-nine 
wolves moving fiercely and close together into a glass wall. The wolves are supposed to collide in the invisible wall; they fall, stand up, and return to their origin to start the cycle again and again.

- "The Tunnel House" by Dan Havel and Dean Ruck. They materialize the possibility of recycling old structures into new forms of interpretation and interaction. Two old buildings were to be replaced by a new built project, so a couple of months before the demolition, they created this installation. The wood from the old building is used to build a wooden tunnel. The result is people in the neighborhood transiting through the tunnel.

- "Swimming pool" by Leandro Erlich. The artist represents the possibility of two parallel worlds in which two types of spectators emerge through the simulation of a swimming pool. The public is an active participant; whether they appear to be inside or outside the pool, two different perspectives are creating while playing with the participants' imagination and sensations.

Previous studies suggest that alternative cultural media represent interesting ways of communicating science.

While designing the installation, the authors focused on creating an installation that would allow each participant to use their imagination to connect scientific facts with everyday objects. The authors decided to dedicate the installation to the environmental deterioration topic, due to the strong campaigns on sustainability and environmental care that the university has carried on the last couple of years. Therefore, the installation conceptualizes important facts on environmental deterioration.

The art installation represents the actual relationship between nature and humankind in the never-ending pursuit of progress. The structure of the installation represents this quest; the objects that constitute it symbolize the resources and products that materialize our footprint. Because of this, the installation is assembled following a specific procedure; to represent the cycle and final outcome that inevitably transform the resources we take along the way.

\section{Selection of participants}

The sample consisted of 36 students enrolled in the Communication and Digital Media Bachelor in their second year of university attending the Contemporary Art course $^{26}$. The mean age of the participants in this sample was twenty-one years of age. Due to the nature of their degree, they hadn't taken any science course in the university.

In further studies we suggest the inclusion of senior students and master students in the art installation design process. Previous to the art piece creation it would be important to present a couple of lectures focusing on the above mentioned literature in which the public participation model, the AEIOU approach, and experiential learning, become the theoretical basis and prepare students to design an art installation. 


\section{Setting for the activity}

The activity took part in two adjacent settings: a classroom and a courtyard. It was programmed for the end of the semester, a couple of days before the final exams. This would guarantee a larger number of students attending the university and a greater audience while assembling the art installation. Since it is important to involve the non participating audience as an active spectator, the activity was programmed for 11:00am, the time of day with more students in the university.

The courtyard was 20 meters by 15 meters. The area considered a space to store the materials that would be used in the installation and enough space that would allow the participants to move freely without any obstructions. The classroom was the setting for the control group; it allowed closeness to the art installation group and at the same time provided a quiet environment to concentrate for the activity.

\section{Stimuli Development}

Due to the fact that the activity is embedded in the Contemporary Art course, the students were advised that they would be participating in the creation of an art piece at the end of the semester. Two classes before the activity they were asked to bring two pairs of shoes and comfortable clothing since the activity would take place in one of the courtyards of the university.

The day of the activity, participants were divided into two groups $(\mathrm{N}=18)$. One group, or the "factual group", was exposed to a list of environmental deterioration facts and the other group, or "art installation group", participated in the creation of the art installation. Both groups were requested to answer the Measures immediately after finishing the activity. The Measure was applied again two months later.

During the first session, the factual group was exposed to a list of ten scientific facts concerning environmental deterioration. After ten minutes a questionnaire was handed to them and they had fifteen minutes to complete it. The second group assembled an art installation following a specific procedure, immediately after been exposed to the ten scientific facts.

To assembly the art installation the group was divided into 3 teams: green, black, and yellow. Each one of them had a specific task during the construction process. A procedure sheet and a diagram (figure 2) were given to each team describing the assembly process, as well as each one's responsibilities in the process. After the installation was completed they were asked to answer the same questionnaire, as the factual group. 


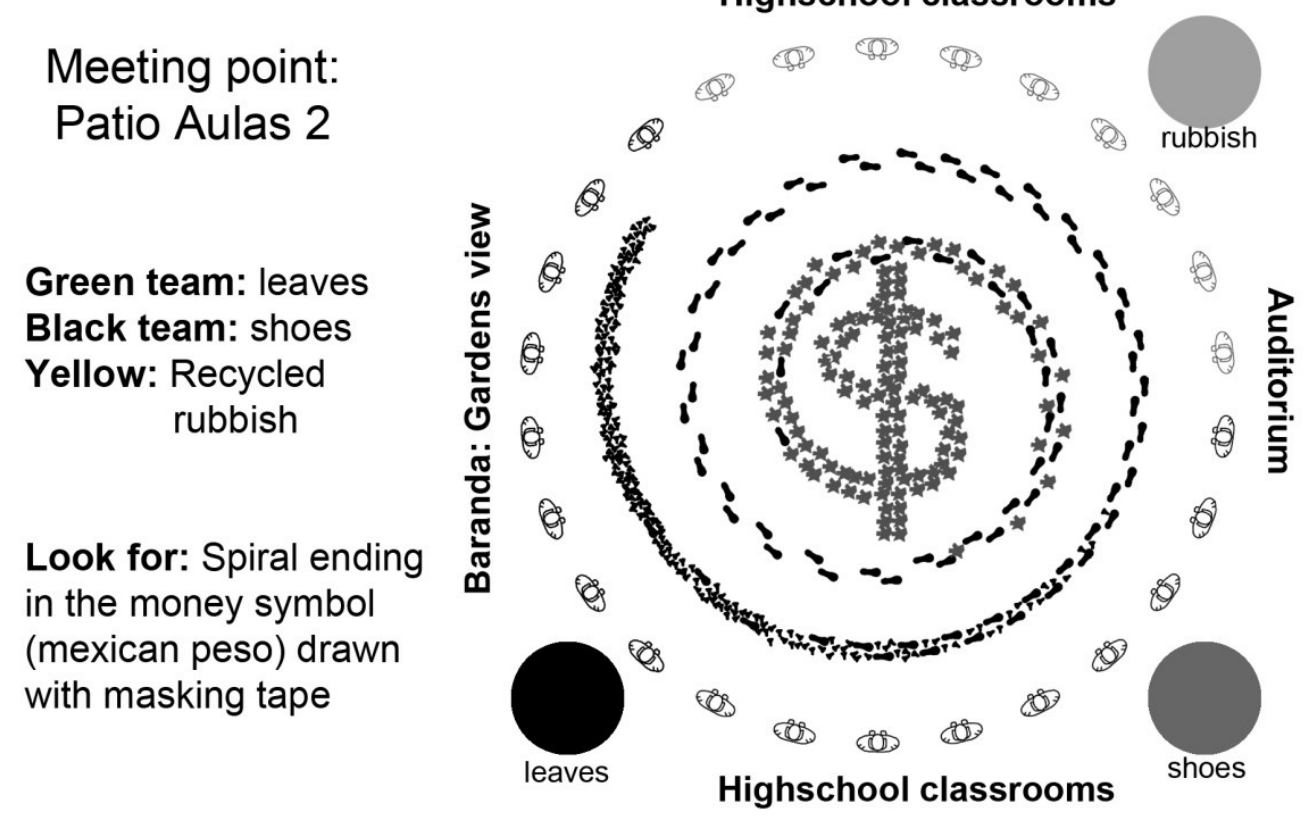

Figure 2. Diagram presented to each team during the assembly process.

These were the environmental facts to which students were exposed. ${ }^{27}$

1. The Earth was formed 4.5 millions of years ago.

2. The first humans appeared 2.5 millions of years ago during the Paleolithic period.

3. Since the first half of the XX century, the human being has been considered the greatest super-predator inhabiting the planet Earth.

4. We have finished with the $80 \%$ of forests on this planet.

5. Each person produces 2 kilograms of rubbish every day.

6. If every country would consume the way the United States of America does, we would need 5 planets to get enough resources.

7. In the past 30 years, we have consumed $33 \%$ of the natural resources on the planet.

8. The economy we have created, demands us to consume. Buying has become a ritual to achieve spiritual satisfaction.

9. The process of buying things is resumed in 5 main steps: Extraction production - distribution - consumption - disposal.

10. It is essential to consider new green technologies to create sustainable environments.

In the case of the art installation group, the assembly procedure was provided in written format, having the possibility of reviewing it during the activity. These following steps were given to each team. ${ }^{28}$

1. (Everyone) Arrival to the meeting point. Leave your shoes in the black corner. In the courtyard, you will find a specific shape made out of masking tape. 
Recognize it and form a circle around it of approximately 10 meters of diameter. Each team will remain together and will pick a leader. Identify the location of the pile of leaves, the recycled garbage, and the shoe pile in the diagram that has been provided.

2. (Green team) Each team member will pick as much leaves as possible from the leaves pile. They will be placed as shown in the diagram. When you are done, the leader will raise his/her hand and the team will return to the circle.

3. (Black team) Each integrant will pick several pairs of shoes from the shoe pile. They will be placed as shown in the diagram. When you are done, the leader will raise his/her hand and the team will return to the circle.

4. (Yellow Team) Each integrant will pick several cans and bottles from the recycled garbage pile. They will be placed as shown in the diagram. When you are done, the leader will raise his/her hand and the team will return to the circle.

5. (Everyone) Make sure the figure is exactly the same as shown in the diagram; make any adjustments if necessary.

6. End of the activity. Refer to your instructor to complete a small questionnaire.

This study was divided into two sessions. During the first session the stimuli were applied to both groups. For the factual group, the stimulus was the list with environmental deterioration facts, and for the art installation group was the list of facts continued by the creation of the art installation. In both cases, the questionnaire was applied immediately after the stimuli.

The questionnaire incorporated 4 different memory tasks to fulfill the requirements of the RIRC Method. The same questionnaire was applied to the factual group and to the art installation group during the first session. Special attention was driven towards the performing conditions, assuring that both groups would answer the questionnaire at the same time of day in one of the university's classrooms.

One question involved the free-recall task (Retell): Mention all the facts that you can recall from the given information. A five-item list of the most important concepts that enclosed the given scientific facts was established. The maximum score for this task was 5.

For the recognition task (Identify), three multiple-choice questions were designed. Therefore, the maximum possible score for this task was 3 . In order to have an optimal answer the participant should be able to identify the mentioned fact from a series of possible options.

For the recall (Remember), two types of questions were considered: short answer and fill in the blank. This kind of tasks helps to measure the information that can be produced using explicit memory. The maximum possible score for this part was 4 .

Question number nine refers to the procedural knowledge task (Contextualize). The objective of this question was to create a different context, and measure the ability of the participants to apply learned facts in a specific situation. This task is connected to opinion-forming and gaining consciousness.

An extra question concerning also procedural knowledge was applied to the art installation group. Its purpose was to identify the ability of participants to relate scientific facts to material, forms, and colors in the installation. This question was 
designed to explore future research opportunities and interpretation outcomes, but was not considered for the statistical analysis. Most art installations intend to provoke an emotion, feeling or sensation among the public. The last question explores the art installation's capability of allowing different kinds of response and interpretations among the participants. Since the shapes, forms, colors, and materials used in the installation conceptualize scientific information, they become open-ended statements, ${ }^{29}$ in which each participant can draw its own interpretations throughout a unique experience. How participants comprehend the installation helps us understand how they become engaged to new scientific knowledge.

The second session was developed two months later. The same questionnaire was applied to both groups but this time without the stimuli in order to measure the amount of scientific knowledge that had been retained in the long-term memory for the last two months. ${ }^{30}$

\section{Statistical methodology}

In order to determine precise estimates of the chosen population, a probabilistic sampling was designed. In this case, the population comprehends current undergraduate students enrolled in the School of Humanities and Social Sciences of a university in Mexico City. ${ }^{31}$ Given the total population of 1879 students, then:

$\mathrm{N}=$ size of the population: 1879 students

$\bar{y}=$ average value of a variable $=1$, one student

$\mathrm{Se}=$ Standard error of .05 , was determined during the research

$\mathrm{V}^{2}=$ population variance (square of standard error)

$\mathrm{S}^{2}=$ sample's variance (probabilistic occurrence of $\bar{y}$ )

It was established a standard error $\leq .05$ and occurrence of .09 , therefore:

$$
\begin{gathered}
n^{\prime}=\frac{.09}{.0025}=36 \\
n^{\prime}=\frac{n^{\prime}}{1+\frac{n^{\prime}}{N}}=\frac{36}{1+\frac{36}{1879}}=36
\end{gathered}
$$

The analysis stated a sample of minimum 36 students which were divided into "factual group" and "art installation group".

Statistical analysis was used with the aim of validating the stimuli results. The Ttest analysis was implemented to compare means among groups and to recognize statistically significant relations among variables. Analysis of variance (ANOVA) was used to verify the statistical effect of the independent variable (art installation participation) in the dependent variable (long-term memory) through the means analysis in session 1 and 2 in both groups. 


\section{Results}

During the first session there was no significant difference between the overall outcomes of the factual and art installation group.

Even though the means of the factual group $(\mathrm{M}=10, \mathrm{SD}=2.89)$ were higher than the ones of the art installation group $(\mathrm{M}=8.76, \mathrm{SD}=2.54)$, the T-test indicates that there is not a significant statistical difference, $t(32)=1.32, p<.05$, in the retained information between the factual and art installation groups. During this session, however, the standard deviation shows that the art installation group had a more homogeneous performance.

In the second session we could observe differences in the amount and quality of scientific information that was retained. During this session the art installation group had better results in the four tasks $(M=10.82, S D=2.48)$ than the factual group $(\mathrm{M}=6.82, \mathrm{SD}=2.90)$ (table 1$)$. The T-test indicates that there is a statistical significant difference, $t(32)=4.32, \quad p<.05$, between the overall results of the art group installation and the factual group in session 2. The overall standard deviation shows that the art installation group had a more homogenous performance.

\begin{tabular}{|c|c|c|c|c|c|c|}
\hline \multirow{7}{*}{$\begin{array}{l}\overrightarrow{\widetilde{\sigma}} \\
\bar{w} \\
\bar{w} \\
\tilde{w}\end{array}$} & & Retell & Identify & Remember & Context & Total \\
\hline & \multicolumn{6}{|c|}{ FACTUAL GROUP } \\
\hline & Mean & 2.88 & 2.35 & 3 & 1.76 & 10 \\
\hline & $\begin{array}{l}\text { Standard } \\
\text { deviation }\end{array}$ & 1.654 & 0.786 & 1.118 & 0.752 & 2.894 \\
\hline & \multicolumn{6}{|c|}{ ART INSTALLATION GROUP } \\
\hline & Mean & 2.29 & 2.29 & 2.59 & 1.59 & 8.76 \\
\hline & $\begin{array}{l}\text { Standard } \\
\text { deviation }\end{array}$ & 1.687 & 0.686 & 0.939 & 0.618 & 2.538 \\
\hline \multirow{6}{*}{$\begin{array}{l}N \\
\tilde{0} \\
\text { w } \\
\tilde{w} \\
\tilde{n}\end{array}$} & \multicolumn{6}{|c|}{ FACTUAL GROUP } \\
\hline & Mean & 1.53 & 1.65 & 1.88 & 1.76 & 6.82 \\
\hline & $\begin{array}{l}\text { Standard } \\
\text { deviation }\end{array}$ & 1.84 & 0.86 & 0.86 & 1.03 & 2.9 \\
\hline & \multicolumn{6}{|c|}{ ART INSTALLATION GROUP } \\
\hline & Mean & 3.24 & 2.59 & 2.71 & 2.29 & 10.82 \\
\hline & $\begin{array}{l}\text { Standard } \\
\text { deviation }\end{array}$ & 1.89 & 0.94 & 1.05 & 0.85 & 2.48 \\
\hline
\end{tabular}

Table 1. Specific task results of the factual group and art installation group during session 1 and 2. 
In the case of the factual group we observe that over time the retained information is lost, especially in the tasks that measure explicit memory. In this sense the factual group experienced a decrease in the overall mean from 10 in the first session to 6.82 in the second session. In the procedural knowledge there were no statistically significant differences between the results in session 1 and 2 of the factual group.

The art installation group showed an improved performance in the second session. For the explicit memory tasks, the T-test shows a statistical significant difference, while $\mathrm{n}$ the implicit memory task the statistical result remains the same (figure 3 ).

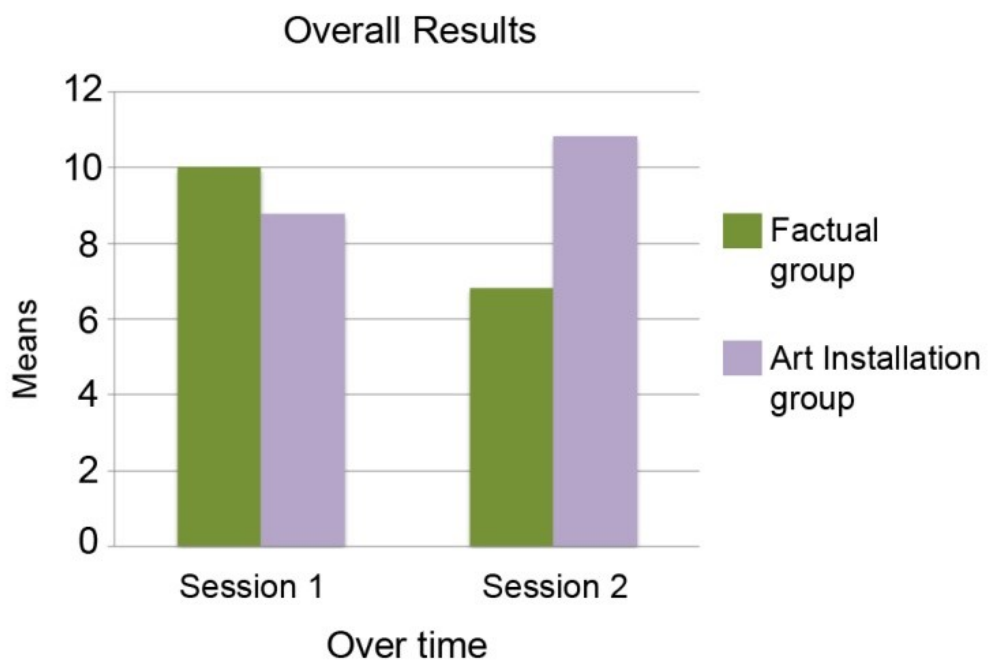

Figure 3. Overall results of the factual and art installation group during session 1 and 2.

A final statistical test was conducted in order to support the positive effect of participating in an art installation to retain scientific knowledge through time. The ANOVA test indicates that there is a statistical significant effect of participating in an art installation, $F(3,64)=7, p=.05$. It confirms that the independent variable (participation in an art installation) increases the amount of retained scientific facts in participants.

\section{Discussion}

The general results show an improvement in the performance of the art installation group, while the number of optimal answers diminished in the factual group (figure 4). This outcome may have been produced by an exchange of information among the participants between the sessions; that is, they commented on the event. 


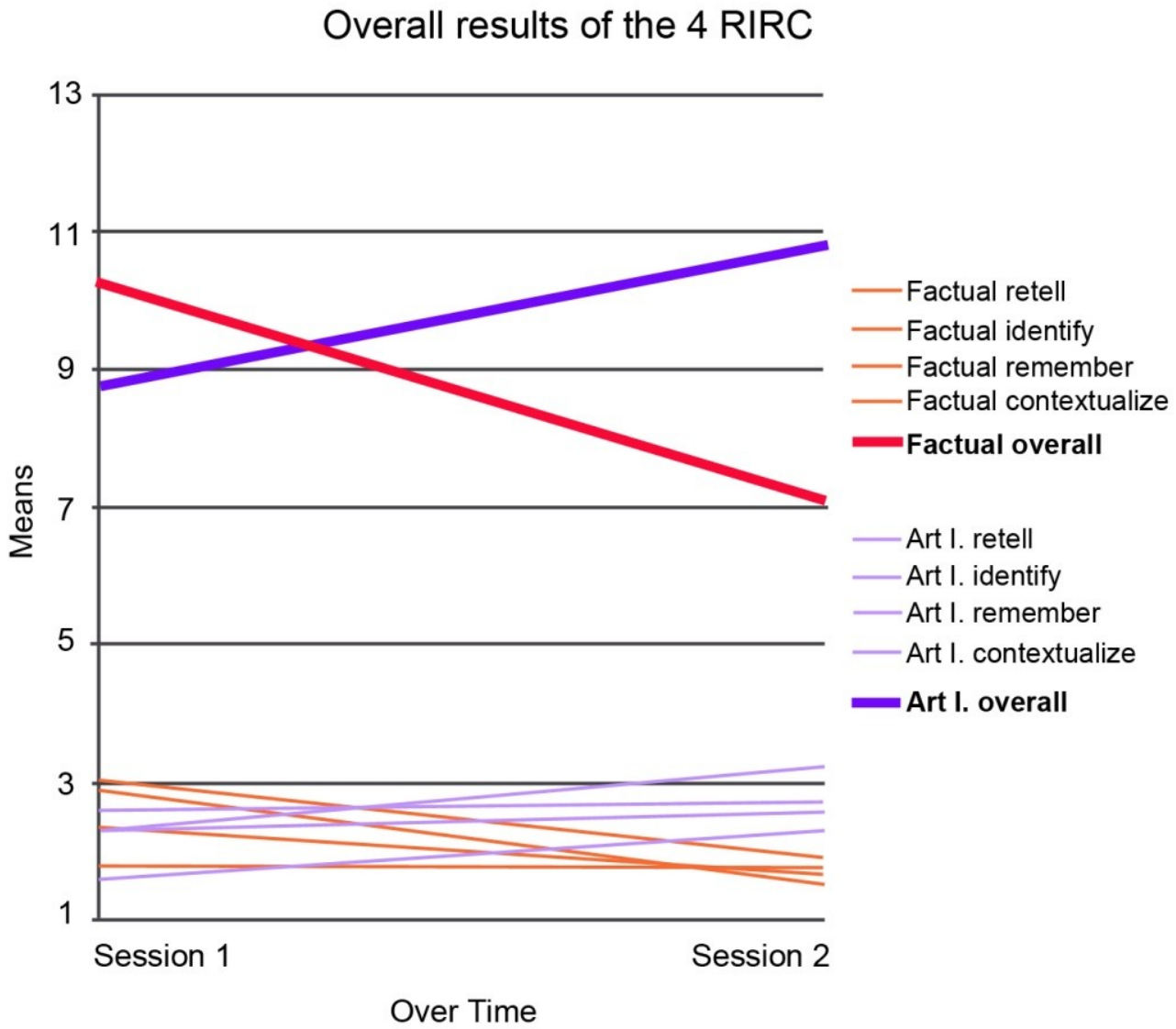

Figure 4. Specific results show an improvement in the performance of the art installation group over time.

The fact that the students took the time to comment and discuss the activity suggests that art installations create interest in the public. After the second sessions, we received mails from four participants wanting to know more about the activity and their results. Two of them were concerned with their final outcome and wanted to know if they did well. One of them wanted to know if there were photographs of the activity. The last participant wanted to know if the activity would be repeated at some point and if he could ask friends to join him.

This couple of comments and interests make us believe that people understood and interiorized the installation assembly in different ways. The questionnaires give the idea that there might be a score being evaluated and therefore appeal to the academic structure. In the other hand, wanting to have empirical evidence such as photographs, suggests that the participant enjoyed the activity and that an emotional bridge was created linking scientific facts and an emotional response. Finally, having a participant that wanted to invite more friends proves that the assembly of an art installation also strengthens community bonds and helps consolidate the sense of identity. ${ }^{32}$ According to these effects, art installations proved to be an interesting and enjoyable way to acquire scientific knowledge. 
The findings in this study match the Negrete and Lartigue outcome ${ }^{33}$ with regard to communicating scientific knowledge via narratives. Those results suggest that science can be learned through literary stories. Their findings suggest that participants retain narrative information for longer periods than do participants receiving factual information. Communicating science through narratives constitutes an accurate, memorable, and enjoyable way to transmit scientific information. In the same way, art installations have demonstrated to be a reliable medium to communicate scientific knowledge.

Also a review conducted by ECSITE (2008) on science and technology museums, zoos, aquaria and science centers suggest that there is evidence that interactive science exhibitions increase visitors knowledge and understanding of science and that these experiences provide memorable learning as well as long lasting impact on attitudes and behavior.

According to this survey also interactive exhibitions generate strong emotions, an important element for long term potentiation. It is plausible that both, interactive exhibitions and art installations result memorable because they promote the three elements for long term potentiation: attention, emotion, and rehearsal. Our results suggest that participants were concentrated in the process of constructing the installation (attention), they reported the installation as a fun experience (emotional response) and they kept commenting about the event days after the construction (rehearsal).

An art installation allowed each participant to acquire knowledge in its own specific way through this particular experience. Even though each participant developed a different task while assembling the art installation, results show that the retained information was homogeneous within the group. This suggests that each person apprehends knowledge in a unique way and, although the opinion-forming process might be different, at the end, the sought for results are attained.

Carrying out this investigation resulted in a very insightful experience. The art installation group participants were able to connect facts with the items, forms and colors presented in the art installation. Participants in the art installation group with higher overall results on the four memory tasks demonstrated a better connection of scientific data to art installation concepts and objects.

One of the most important aspects that should be emphasized in this investigation is the ability of art installations to sensitize the participants by experiencing different objects, feeling them, smelling and hearing scientific information at the same time. Different sensations are perceived; for instance, it is not the same to smell green fresh leaves, than to smell recycled cans and plastic bottles. This suggests that involving different senses in the apprehension of new scientific knowledge might enhance awareness and interest among the public. Furthermore, it suggests a new approach to the public participation model of science communication.

The ability of a person to develop consciousness is what gives facts an extra value; it is not just about retaining information, but also of being able to use it in different situations. This research opens interesting questions about future research and variables 
that might be considered in order to explore how the appropriation of scientific knowledge can lead to more engaging activities.

\section{Future Research}

Art installations are one of many forms of art expressions that require public participation. This research opens the possibility of exploring other artistic forms and their effects on the communication of scientific knowledge.

While conducting future research it will be essential to broaden the sample as well as to consider different age groups. The art installation is a late expression of the twentieth century. It is particularly interesting for young people. It will also be important to analyze the effect among adults and older generations.

Nowadays, sustainability and environmental topics are common topics of public discussion. Future research suggests the incorporation of other scientific themes into the creation of art installation in order to assess and compare the results.

Furthermore, in this study the art installation was previously designed, it would be interesting to involve the students in the design process and bring together different perceptions with the aim of communicating the information more effectively to a broader audience. The creation itself of an art piece, demands consolidated knowledge on a topic in order to explore different media and tools to represent it. The better the artist understands an issue, the easier and clearer it will be for him/her to create an art piece.

After analyzing the process the authors followed to create the art installation, we realize that they bring together through meticulous process scientific information, SciCom theory, concepts, materials, technics, and symbolism. This research emphasized the importance of interdisciplinary work in which experts in science communication and artist can only achieve the final goal through a structured communication process.

\section{Conclusion}

Our results suggest that immediately after acquiring knowledge there was no difference between the factual and art installation group in the amount of retained information. However, after two months this study suggests that participating in the creation of an art installation enhances the retention of information in the long-term memory. The results suggest that an experience that promotes experiential learning also promotes the more accurate retention of scientific information over time. The evidence gathered in this research suggests that while information presented with a list of scientific facts loses accuracy and homogeneity over time, the information presented via art installation gains accuracy as well as homogeneity. These results strengthen our belief that art installations help retain scientific information.

The overall results of this study, suggest that art installations do aid in long-term retention of scientific information. In general, there is enough statistical evidence to foster the belief that participation in art installation enhances the retention of scientific 
information in long-term memory. Therefore, the creation of art installations can be considered an interesting method to convey science in an attractive, reliable, and memorable way.

When the communicating process is not only employed as a one-way communication process, but also is participated in by the public, a different kind of awareness is created. The results suggest that the public participation model can be addressed in different ways while also fostering public engagement and understanding.

\section{Notes and references}

1 C.P. Snow (1998), The two cultures, Cambridge University Press, Cambridge, U.K.

2 G. Rowe and J.L. Frewer (2000), Public participation methods: a framework for evaluation, Science, technology, and human values 25(1): 3-29.

${ }^{3}$ B.V. Lewenstein (2003), Models of public communication of science and technology, Pub. Underst. Sci. 16: 1-11.

${ }^{4}$ European Network of Science Centres and Museums (Ecsite) (2008), The impact of Science \& Discovery Centres; A review of woldwide studies, http://www.ecsite.eu/.

${ }^{5}$ G. Cowan (2005), Bridging art and science through the ages, Explorer magazine Argonne National Laboratory.

${ }^{6}$ L. O'Farrell (1994), Education and the art of drama, Deakin University, Australia.

${ }^{7}$ H. Gardner (1983), Multiple intelligences: The theory in practice. Basic Books, New York, U.S.A.

${ }^{8}$ P. Vergo (1989), The new museology, Reaktion Books, London, U.K.

${ }^{9}$ M. Horst (2011), Taking our own medicine: on an experiment in science communication, Science and Engineering Ethics 17(4): 801-815.

${ }^{10}$ See note 5 .

${ }^{11}$ D. Anderson, B. Piscitelli, K. Weier, M. Everett and C. Taylor (2002), Children's Museum Experiences: Identifying Powerful Mediators of Learning, Curator: The Museum Journal 45(3): 213-231.

${ }^{12}$ T.W. Burns, D.J. O'Connor, S.M. Stocklmayer (2003), Science Communication: A Contemporary Definition, Pub. Underst. Sci. 12: 183-202.

${ }^{13}$ L. O'Brian (2000), Learn to remember, Duncan Baird Publishers, Nueva York.

${ }^{14}$ C. Rogers (1969), Freedom to learn, Merrill, Columbus, OH, U.S.A.

${ }^{15}$ E. Tulving (1993), What is episodic memory?, Current Directions in Psychological Science 2: 67-70.

${ }^{16}$ T.J. Shores and L.D. Matzel (1997), Long-term potentiation: What's learning got to do with it?, Behavioral and Brain Science 20: 597-655.

${ }^{17}$ See note 16.

${ }^{18}$ A. Negrete (2008), La divulgación de la ciencia a través de formas narrativas. Divulgación para divulgadores, México, D.F.

${ }^{19}$ A. Negrete and C. Lartigue (2010), The science of telling stories: Evaluating science communication via narratives (RIRC method), J. Media Commun. Studies 2: 98-110.

${ }^{20}$ N. Gough (1993), Laboratories in fiction: science education and popular media, Deakin University, Geelong.

${ }^{21}$ See note 20

${ }^{22}$ P.M. Applebaum (1995), Popular culture, educational discourse, and mathematics, University of New York Press, New York, U.S.A.

${ }^{23}$ M. Weinstein (1998), Playing the paramecium: science education from the stance of the cultural studies of science, Educational Policy 12(5): 484-506.

${ }^{24}$ J. Weaver (1999), Synthetically growing a post-human curriculum: Noel's Gough curriculum as a popular cultural text, Journal of Curriculum Theorizing 15(4): 161-169. 
${ }^{25}$ E.E. Nunan and D. Homer (1981), Science, science fiction, and radical science education, ScienceFiction Studies 8: 311-330.

${ }^{26}$ The study started with a group of 25 students, however, some of the students didn't completed session 2. The final results consider 18 students having completed session 1 and session 2 .

${ }^{27}$ The original facts were provided in the students' native tongue: Spanish. For the article's purposes information has been translated.

${ }^{28}$ The original assembly process was provided in the students' native tongue: Spanish. For the article's purposes information has been translated.

${ }^{29}$ See note 9.

${ }^{30}$ The second questionnaire was applied via email, giving strict instructions not to consult any type of bibliography. In further research it is suggested to apply the second questionnaire under the same conditions as the first one.

${ }^{31}$ Instituto Tecnologico de Estudios Superiores de Monterrey, Mexico City, Mexico.

${ }^{32}$ K.M. Ellenbogen (2003), From dioramas to the dinner table: an ethnographic case study of the role of science museums in family life, doctoral dissertation, Faculty of the Graduate School of Vanderbilt University, Nashville, Tennessee, U.S.A.

${ }^{33}$ See note 19 , outcome.

\section{Authors}

Patricia Rios was born in 1983 in Mexico City. She earned a degree in Architecture from ITESM and is a Ph.D. Candidate in the same institution. She is co-founder of the workshop C-3: City and Community Collective, a group of architects and urban planners dedicated to integrate the participatory process in the design of the city. Her work as a researcher includes topics such as: multidisciplinary approach towards the sustainable city, children as agents of future change, participatory design and science communication, and the science museum as a public space. She is currently teaches Urban Project and Integrated Projects III at ITESM Campus Queretaro.

E-mail: riospaty@gmail.com.

Dr. Aquiles Negrete Yankelevich is a professor of Science Communication in UNAM. He received an MSc in Resource Management from Edinburgh University U.K. and a Ph.D. in Science Communication from the University of Bath U.K. He is author of a number of scientific papers and various works in fictional literature. In 2009 he published the book "So, What Did You Learn from the Story? Science communication via narratives". His current research interests are centred on science communication via cultural media. E-mail: aqny@unam.mx.

How TO CITE: P. Rios and A. Negrete Yankelevich, "The object of art in science: science communication via art installation", JCOM 12(03) (2013) A04. 